\title{
La lecture, les formes et la vie : Entretien avec
} Marielle Macé

\section{Marielle Macé, Raphaël Baroni et Antonio Rodriguez}

\section{(2) OpenEdition}

\section{Journals}

Édition électronique

URL : http://journals.openedition.org/edl/619

DOI : $10.4000 /$ edl. 619

ISSN : 2296-5084

Éditeur

Université de Lausanne

\section{Édition imprimée}

Date de publication : 15 mars 2014

Pagination : 165-180

ISBN : 978-2-940331-34-5

ISSN : 0014-2026

\section{Référence électronique}

Marielle Macé, Raphaël Baroni et Antonio Rodriguez, « La lecture, les formes et la vie : Entretien avec Marielle Macé », Études de lettres [En ligne], 1 | 2014, mis en ligne le 15 mars 2017, consulté le 19 décembre 2020. URL : http://journals.openedition.org/edl/619; DOI : https://doi.org/10.4000/edl.619 


\section{LA LECTURE, LES FORMES ET LA VIE

\author{
Entretien avec Marielle Macé \\ par Raphä̈l Baroni et Antonio Rodriguez
}

Dans son dernier livre, Façons de lire, manières d'être, Marielle Macé replace l'expérience de lecture à l'intérieur d'une vaste "stylistique de l'existence»; elle établit un lien entre cet élargissement de la question du style aux formes de la vie, et ce que nous pouvons attendre des livres: la lecture lui apparaît comme l'une de ces conduites par lesquelles, quotidiennement, nous donnons un aspect, une figure, des formes, des rythmes, quelque chose comme "un style» à notre existence. Son ouvrage part d'une réinterprétation des rapports entre la littérature et la vie; il pose que c'est dans la vie elle-même que les œuvres se tiennent, déposent leurs traces et exercent leur force. Il n'y a pas d'un côté la littérature, et de l'autre la vie; il y a au contraire, dans la vie elle-même, des formes, des images, des styles d'être qui circulent entre les sujets et les œuvres, qui les exposent, les animent, les affectent, les transforment (ou les laissent indifférents). En sorte que les formes littéraires se proposent dans la lecture comme de véritables phrasés de la vie, engageant des conduites, des rythmes, des puissances de façonnement, des valeurs pratiques. Dans l'expérience ordinaire et extraordinaire de la lecture, chacun peut alors se réapproprier son rapport à soi, à son langage, à ses possibles, à ses modes d'être, et la littérature apparaît comme le lieu où se médite ce qu'il entre de formes dans la vie.

Raphaël Baroni (RB) : Marielle Macé, dans votre dernier ouvrage ${ }^{1}$, vous abordez la lecture littéraire sous un angle original, que l'on pourrait apparenter à une sorte de "stylistique existentielle». Ce rapport entre façons de lire et manières d'être (dont je pense qu'il est important de souligner le pluriel) a notamment pour enjeu de retisser des liens entre

I. Façons de lire, manières d'être, Paris, Gallimard, coll. «NRF-Essais», 2011. 
l'expérience esthétique et la vie, dans la plus large extension que ce terme peut avoir.

Marielle Macé (MM) : En effet j'ai essayé d'aborder dans cette recherche la lecture, ou le rapport aux œuvres, à travers une hypothèse plus vaste: celle d'une stylistique de l'existence. Une "stylistique de l'existence", c'est-à-dire une stylistique élargie à la vie elle-même, autrement dit une attention aux formalités du vivre, à tout ce qu'il entre de formes (de rythmes, de gestes, de manières de faire ou de dire..., bref, de "comment») dans nos pratiques. Je différencie fortement cette stylistique de l'existence d'une "esthétique de l'existence", qui, elle, viserait la façon dont les sujets embellissent leur propre vie, se traitent comme des œuvres d'art, concertent leurs apparences, choisissent leur identité, espèrent une "vie en beau", comme le disait avec violence et ressentiment le héros du "Vitrier» de Baudelaire (la clarification de cette différence est d'ailleurs le point de départ de l'essai que je prépare actuellement). De ce point de vue, le pluriel des "manières d'être » est en effet très important; il indique que l'on peut être attentif à toutes les formes, à tous les «tours» que prend la vie, et pas seulement aux formes prisées, reconnues, valorisées.

C'est dans ce cadre général, et avec cet espoir d'une véritable ouverture éthique de la question du style, que je me suis efforcée de repenser les rapports, comme on dit, entre "la littérature et la vie». Ainsi que vous l'avez rappelé dans la présentation, je ne crois pas qu'il y ait d'un côté la littérature et de l'autre la vie, ou les vies, et qu'il faille travailler à les relier; non que je veuille assimiler "la vraie vie" à la littérature, bien au contraire, mais parce que je crois que les ouvres nous parviennent au cœur de notre vie, avec nos questions, avec nos attentes. La lecture n'est pas un moment autonome, isolé; elle prend place à l'intérieur d'une "aventure esthétique» permanente, qui engage tous nos gestes, toutes nos rencontres, toutes les formalités du vivre. Il m’a semblé important de souligner cela, de manifester ce grain stylistique permanent de la vie.

Et vous dites bien qu'il s'agit de retisser des liens. Certes, pour lire on commence le plus souvent par se retrancher, et même par se défaire du monde environnant et de ses exigences - Proust a décrit cela patiemment, magnifiquement. La lecture sépare physiquement, et aussi, ou surtout, socialement: on décide de suivre une ligne qui reste en partie invisible à autrui, on acquiesce à un certain silence, à un autre état de 
conscience, on se laisse assiéger par une voix extérieure qui refaçonne entièrement, pendant un temps, notre bande-son intérieure. Le livre est comme un habitat concurrent de notre habitat ordinaire - Quignard dit fortement qu'un lecteur se retrouve "seul chez son livre», comme s'il avait à demeurer tout à coup dans une maison étrangère.

Mais dans ce retranchement, on ne tourne pas le dos au monde. A vrai dire, on passe son temps, en lisant, à lever les yeux de son livre; Barthes avait une belle formule pour décrire cette respiration entre le livre et le monde, ce battement assez particulier de la lecture: "Ne vous est-il jamais arrivé, disait-il, de lire en levant la tête?», non par désintérêt, mais par afflux d'idées, d'associations, de réactions, de mises en rapports - "c'est comme», "ce n'est pas comme»... Non seulement on ne quitte pas la vie en lisant, mais ce qui se passe dans la lecture a, au moins en droit, un avenir dans cette vie; on prépare des pensées, des souvenirs, des façons de dire et de se rapporter aux autres, on augmente ses capacités d'attention et ses manières de voir, on module son propre accès au monde, on essaie d'autres liens, d'autres manières. Parfois d'ailleurs on souligne intérieurement telle ou telle phrase, telle ou telle situation qui pourront devenir un nouvel "équipement» d'expérience ou de pensée - on prépare une citation; et l'on ne sait pas forcément comment elle entrera en résonance avec le monde et trouvera un emploi, si je puis dire, dans notre existence, mais on redirige déjà la lecture vers la vie.

C'est ce qui m’a fait prendre d'emblée mes distances avec les modèles sémiotiques, tout simplement parce que l'échelle (et l'absence de temporalité) à laquelle ils saisissent la lecture (comme une petite performance autonome, séparée, qui commencerait à la première page du livre et serait achevée une fois le livre refermé) n'est pas celle qui me semble donner l'essentiel de sa portée à la lecture. La plupart des sémiotiques de la lecture me paraissent figurer celle-ci comme un cheminement clos sur luimême, selon une sorte d'imaginaire ferroviaire où le petit wagon qu'est le lecteur, engagé sur les rails narratifs, dirigerait essentiellement son activité vers une anticipation des pistes du récit, formulerait des hypothèses qui se trouveraient ensuite confirmées ou démenties par le texte, et s'orienterait ainsi vers un futur qui est, avant tout, la fin de l'histoire. Le livre refermé, l'affaire serait faite. Pourtant, le futur visé par le lecteur n'est pas seulement la fin de l'histoire, c'est aussi le sien; les pistes envisagées par le lecteur ne sont pas les voies du texte, ce sont aussi les orientations de son monde; les aiguillages auxquels il se confronte ne sont pas 
les virtualités narratives infirmées ou confirmées par la suite du récit, ce sont aussi ses choix; bref le temps de la lecture dépasse largement celui d'une réalisation sémiotique ponctuelle séparée de la vie quotidienne. A mes yeux, cela supposait aussi de m'écarter d'une conception de l'acte de lecture comme "comblement de lacunes": je ne crois pas que les livres nous mettent essentiellement en présence de blancs ou de vides à combler (des creux dans l'histoire par exemple, ou ce que la théorie a parfois appelé des «indéterminations»), mais avant tout en présence de "pleins", de formes fortes, éloignées, auxquelles nous devons faire place en nous-mêmes. J'ai donc plutôt cherché à comprendre ce qui pouvait se jouer au long d'une vie entière de lecteur, dans la suite de transformations et de pertes qui adviennent au contact d'œuvres successives: la lecture dans la vie donc, et tout au long d'une vie. Le désir de reconnaître l'activité du style à même l'existence constitue pour moi le cadre général de ce changement d'échelle, et de ce changement de focale: je voulais comprendre comment des formes pouvaient faire leur chemin à l'intérieur de nous, déposer des traces mentales, et par conséquent constituer des ressources.

RB : Avez-vous, par ailleurs, l'impression de rejoindre la position de Todorov qui, dans La Littérature en péril, enjoint les enseignants à sortir d'une approche trop «technicienne» des textes pour retrouver une forme de lecture humaniste?

MM: Rejoindre cette position? Pas tout à fait, ou pas exactement ainsi ; car c'est prioritairement aux formes littéraires que j'ai cherché à restituer leur valeur existentielle, anthropologique, ou sociale; autrement dit, c'est à tout ce que peut une forme, in fine, que je m'intéresse; cela implique de trouver beaucoup d'intérêt à la partie "technique» de l'analyse littéraire; mais aussi, vous avez raison, de la diriger autrement. Formes littéraires, formes de vie: c'est ce rapport, ce miroitement ou cette tension, en tout cas ce travail réciproque qui m'a importé. Et c'est l'ampleur de cette articulation entre les formes littéraires et les formes de la vie que j'ai cherché à décrire; encore une fois, non pas pour suggérer que la vraie vie est la littérature, mais pour dire que toute vie se joue dans des formes, et que l'un des intérêts des œuvres d'art, de ce point de vue, est qu'elles savent prendre en charge, en responsabilité, cet aspect de l'existence. 
Je crois que les styles littéraires se proposent justement, au cours de la lecture, comme des formes de vie: ils engagent des manières d'être, des possibles, ils nous conduisent à intervenir sur nos manières d'être, et à nous éprouver nous-mêmes comme "possibles». Dans mon expérience de lectrice, par exemple, les inventions grammaticales d'Henri Michaux ont été des leçons formidables: j’ai été emportée par la manière dont Michaux façonnait des figures inédites de la subjectivité, réfléchissait en poète au rapport complexe que chacun de nous entretient avec soimême, à la façon dont nous nous divisons intérieurement, dont nous nous construisons ou dont nous nous défaisons, dans son seul usage des formes verbales de la réflexivité: "Harmonise tes détériorations", "Ne laisse pas "toi" te gagner", "Va suffisamment loin en toi pour que ton style ne puisse plus suivre»... Voilà une grammaire troublante de la vie, du devenir: qu'est-ce qui se divise ici ? Où passe la frontière entre "toi " et «ton style»?

Cela suppose de se pencher sur des faits de formes, de vouloir y rendre les élèves sensibles, de tenter de leur donner les moyens, avant tout, de les percevoir, et d'accepter que ces phénomènes aient un intérêt, un enjeu, avant même qu'on ne s'attelle à les commenter. Mais cela suppose, aussi, d'entrer dans une démarche herméneutique, qui justement ne se satisfait pas d'une interprétation «techniciste» des faits de forme; et c'est là sans doute que ma démarche rejoint tous les travaux issus de la philosophie morale.

Antonio Rodriguez (AR) : La didactique s'est notamment construite contre la figure d'autorité dans l'enseignement. Vous définissez la lecture comme "une conduite, un comportement plutôt qu'un déchiffrement». Comment le travail d'éducation devrait-il se réaliser alors? Faut-il apprendre à se conduire face à un texte? Comment voyez-vous la rencontre des subjectivités dans une classe?

MM: Je ne pense pas qu'on ait à "enseigner des conduites", autrement dit à indiquer ce qui devrait advenir dans la lecture; mais, de façon plus circonscrite et peut-être plus transmissible, à donner aux élèves les moyens de percevoir des dynamiques esthétiques, à leur donner les moyens de s'intéresser à elles et de les interpréter. Dans ma propre expérience d'élève, l'école a été ce lieu et cet ensemble de discours qui m’ont rendu perceptibles (visibles, audibles) et surtout disponibles ces formes 
dont je crois aujourd'hui qu'elles sont l'essentiel de l'action littéraire. Seule l'école pouvait me donner cet accès, me donner cette attention, et c'est elle aujourd'hui qui justifie ma pratique. Cet accès aux formes, à des configurations douées d'effets, c'est aussi, socialement, l'accès à une puissance qui ne soit pas un pouvoir, et à une admiration qui ne soit pas un assujettissement; les œuvres m'apparaissent comme ces configurations dotées d'autorité, mais "dénuées d'arrogance», comme l'a dit un philosophe de la technique.

Même si je ne cesse d'enseigner, j'ai tout à apprendre en matière de théorie didactique, et c'est avec bonheur que j'ai mesuré, notamment grâce aux travaux de Gérard Langlade, mais aussi à ceux de Jean-Marie Schaeffer, ou à des échanges avec Simone Giusti, que ma démarche pouvait consoner avec certaines interrogations pédagogiques. Mais de ce point de vue, je ne peux donner que ce que j'ai, car je n'ai pas de légitimité à tenir un discours prescriptif sur l'enseignement. Ma réflexion sur la lecture n'indique pas de méthode, et surtout pas une méthode qui prétendrait inviter la vie dans la classe, ou l'y remettre - comme si elle n'y était pas déjà. Mais sans doute elle tourne nouvellement l'attention vers ce "sujet" qui est le foyer (mais aussi la limite) nécessaire de toute expérience esthétique, et qui ne peut pas ne pas l'être, et c'est peut-être cela qui fournit des pistes de réflexion pédagogique. Elle ne propose pas non plus d'exercices; peut-être nomme-t-elle, de façon plus générale, moins pratique, mais pas moins engagée, des motifs à lire et des motifs à transmettre la littérature (c'est l'écho que j'en ai reçu de la part des enseignants, ou des bibliothécaires) : j'ai cherché à penser jusqu'où peut aller la lecture, à définir ce que la lecture peut atteindre chez un sujet. Ce n'est là ni une méthode ni un exercice, car je ne pense pas que ce "sujet" définisse une posture ou un comportement que l'élève serait en devoir, ou en mesure, d'adopter en classe; ce sujet reste en partie invisible, intime, conflictuel; il se constate au bout d'une vie; il a donc à voir avec l'école comme avec ce qui s'en défait. Et sans doute nous n'avons pas, comme enseignants, à chercher à le faire comparaître sur la scène scolaire ou universitaire; mais l'on peut déjà montrer tout ce que la littérature dit à son propos.

Je crois que, jusque dans les configurations les plus collectives, les plus instituées, la lecture se vit pour partie à la première personne; elle arrive à des individus, requiert des individus pour advenir. La plupart des réflexions sur la lecture contournent cet aspect; il m’a semblé pourtant 
que ce fait que la lecture n'arrive qu'à des individus n'était pas un obstacle à la réflexion, mais pouvait en devenir l'horizon. On peut d'ailleurs dire que la lecture met même en jeu, en chacun, ce fait d'être un individu parmi d'autres, de le devenir ou d'y échapper. C'est un point qui peut être mal compris, car lorsque l'on entend le mot «individu" on a tendance à songer, dans un vocabulaire grevé de libéralisme, à son autonomie, à son originalité, à sa suffisance. Ce n'est pas ce que je voulais viser; ce qui m'intéressait était ce que les philosophes, les anthropologues, les sociologues appellent "l'individuation"; cette notion ne désigne pas un triomphe de la personne (be yourself!), mais le processus de façonnement infini d'un être aux prises avec des forces extérieures, par exemple des œuvres - mais aussi des habitudes, des institutions, des rencontres et des événements de tous ordres; ce processus est fait de déséquilibres et de rééquilibrages, d'aventures et de pertes, car l'individuation ne va pas sans désindividuation. Je n'ai donc pas cherché à décrire la lecture comme une expérience subjective, c'est-à-dire comme quelque chose qui resterait au fond assez impartageable, mais comme une expérience subjectivante et désubjectivante, offrant par conséquent des figures et des occasions complexes, contradictoires, à la compréhension du métier de vivre, si je puis le dire ainsi.

AR: Vous définissez encore la lecture comme un véritable «corps à corps " ${ }^{2}$, où un style se révèle face à l'autre, dans le prolongement de Merleau-Ponty. Ce qui me frappe de mon côté est de constater combien les sensations, les émotions en littérature ne sont pas immédiatement perceptibles, contrairement à nos vies corporelles, mais elles résultent de nombreuses expériences textuelles, voire d'une éducation esthétique, que cherche à initier la scolarité. Pour être sensible aux effets de l'enjambement à la césure chez Baudelaire ou Mallarmé, il faut beaucoup d'expérience. Le «corps» du lecteur n'est-il pas d'abord le fruit d'une éducation qui révèle le style progressivement? En quoi ce corps du lecteur serait-il naturel ou immédiatement donné?

MM: Il n'est évidemment pas donné: lui aussi se fait et se défait tous les jours. Vous avez raison de parler non seulement d'une succession d'expériences nombreuses, dont les leçons se répètent ou sont contradictoires,

2. M. Macé, Façons de lire, manières d'être, p. 23. 
mais surtout d'une "éducation esthétique", et cela confirme ce que j'essayais de dire à l'instant au sujet de ce qui m'est apparu de ma propre formation. C'est une évidence, notamment, pour la poésie.

J'ai par exemple essayé de décrire l'expérience poétique comme une dynamique de déstabilisation et de rééquilibrage rythmiques, par lesquels la poésie met notre tempo intérieur à l'épreuve : elle crée des seuils là où la langue n'en prévoit pas, et par conséquent nous fait hésiter dans notre propre langue, puis nous réassure; de la sorte, elle nous conduit à nous éprouver comme des êtres temporels - à perdre aussi bien qu'à recréer un rythme. Mais cette épreuve rythmique, cette intervention sur nos propres manières de nous tenir dans le temps, n'a lieu que si l'on a été formé, ou autorisé, par exemple, à percevoir les frontières d'un vers, ou à comprendre la duplicité de structure qui existe par définition, dans la poésie, entre la forme syntaxique et la forme métrique. Sans cela, tout simplement, rien de rythmique n'advient - même si bien d'autres choses peuvent advenir (cela explique peut-être aussi que les chercheurs de notre génération aient souvent une approche du cinéma assez différente de celle qu'ils ont de la littérature, simplement parce qu'il n'y avait pas d'éducation aux images dans notre scolarité).

Un exemple, peut-être; j'ai consacré récemment un cours aux Odes funambulesques de Banville, avec des étudiants peu formés à la littérature; lire Banville aujourd'hui, et dans ces circonstances, ce n'est pas évident; mais nous sommes arrivés à quelque chose en entrant en matière avec la théorie du vers et avec l'histoire du vers français; mais aussi avec Mauss, Leroi-Gourhan, Benveniste; je pouvais nommer leurs théories du rythme, et tenter de les approfondir, puisque j'avais affaire à des étudiants de master en sciences sociales; mais je crois qu'il n'est pas déplacé de simplement s'inspirer de l'anthropologie (des formes, du geste, du langage) pour orienter autrement l'étude des formes poétiques, et suggérer quelle portée (historique, esthétique, morale) un équilibrisme de parole apparemment aussi éloigné que celui de Banville peut avoir. Il me semble, de ce point de vue, que l'on doit faire de la philosophie, mais aussi de l'anthropologie ou de la sociologie, en classe de français, tout simplement parce que la littérature en fait déjà, que les textes que l'on présente aux élèves suscitent d'emblée de tels enjeux, et qu'on ne saurait les passer sous silence, ni laisser les élèves seuls avec ces questions. J'ai trouvé intéressant de faire réfléchir des étudiants à la rythmicité de la vie (vie sociale, individuelle, politique) dans le but de tourner leur attention 
vers l'action rythmique de la poésie, vers la façon dont les poètes mettent leur rythme dans le monde.

Si j'ai parlé de "corps-à-corps", ce n'était donc pas tant pour mettre "du corps" (ni même des émotions) dans l'affaire, que pour insister sur la tâche que suppose le fait de rejoindre une forme, d'aller la trouver là où elle est capable d'exercer une certaine force, d'avoir une certaine efficacité; c'est une tâche, car elle s'appuie sur l'étrangeté et sur le lointain des formes, et qu'elle implique d'accepter de faire de la place en soi-même pour leur altérité. C'est pour cela que j'ai souvent insisté sur les moments de difficulté, de refus ou de conflictualité, où un individu (même Sartre, même Michaux) tourne le dos à une expérience, ou ne parvient pas à rapatrier une forme dans le monde de la vie.

AR: Très souvent, pour intéresser les élèves, les enseignants commencent par le «sujet» ou le «thème» actualisé d'un texte. Ils traitent des passions, des choix à réaliser dans une vie, des mœurs ou des idéologies. Or vous considérez le sens d'un texte comme «l'ensemble des événements mentaux causé par un énoncé». Faut-il alors travailler davantage sur la psychologie du lecteur que sur les représentations dans le texte ou la manière de les écrire? Une impression d'accélération ressentie par le lecteur estelle aussi importante que la description commune d'un rythme syntaxique dans le texte? Comment lier les deux choses? Faut-il d'ailleurs les lier?

MM: Là encore, je ne peux parler qu'avec prudence, et en conscience de mon incompétence. Mais je ne pense pas qu'il faille en classe travailler sur la psychologie du lecteur, la démarche me semblerait extrêmement artificielle, et contournée; plus simplement, il me semble qu'il faut... travailler sans relâche sur les énoncés, c'est-à-dire sur des formes singulières. La stylistique, la traduction, la thématique apparaissent ici comme des outils irremplaçables, à partir du moment où l'on vise avec eux autre chose qu'eux. La réflexion récente de Barbara Cassin sur le verbe «entrer", dont elle approfondit l'étymologie, les orientations, les usages, en les mettant immédiatement en rapport avec la capacité d'une communauté de parole à accueillir les exilés (et avec les conditions politiques et économiques faites aux réfugiés), m’apparaît comme un exemple éclatant de ce genre de transmission des effets d'une forme de langage. 
En fait la définition affective du «sens» que vous rapportez n'est pas tout à fait la mienne, c'est celle de Stanley Fish; elle correspond plutôt à une étape dans mon livre, celle des formes de la réclusion et se trouve ensuite dépassée, du moins je l'espère, vers une sortie, et vers l'exigence d'une acceptation de la tâche herméneutique dont je parlais plus haut.

Je ne crois pas que mon étude débouche sur une pédagogie du "ressenti»; mais là encore, je mesure le manque d'information qui est le mien en ces questions. Encore une fois, ce que je décris n'est sans doute pas l'objet de l'enseignement littéraire, ce serait plutôt son horizon, ou l'une de ses justifications: la certitude de l'effectivité des formes, mais aussi le sentiment qu'il faut rendre, culturellement, cette effectivité possible, aider les formes à exercer leur force. De ce point de vue, je ne cherche pas du tout à dévaloriser "l'analyse», par exemple au profit de l'impression, mais à ré-accrocher les formes qui en sont l'objet à des enjeux vitaux; par exemple, pour reprendre le cas de la poésie que je citais tout à l'heure, à rendre possible une compréhension existentielle, sociale, politique, des faits de rythmes. Il y a toutes sortes de textes à mobiliser pour cela: ceux de Baudelaire, de Michaux, de Rilke lorsqu'ils sont en délicatesse avec la scansion de la vie sociale dont ils sont contemporains, qu'ils cherchent à échapper au "rythme des autres", ou au contraire à s'accorder régulièrement au tempo et aux figures temporelles de leur communauté politique; ceux de Rousseau sur la forme du jour (qu'a commentés Jean Starobinski); ceux de Barthes ou de Foucault sur les violences faites aux rythmes de vie; ceux de sociologues contemporains sur les rythmes scolaires, ou sur les rythmes professionnels; ceux des théoriciens du politique, qui héritent ici de Benveniste...

Ce qui s'y manifesterait, c'est que les formes avec lesquelles travaillent les écrivains, et que dégage l'explication de texte elle-même, sont des formes de la vie, des réponses apportées par les textes aux questions que pose la vie elle-même. Et l'on rejoint ici l'effet d'actualisation dont vous parliez au tout début de votre question.

AR: J'aime particulièrement l'inflexion que vous donnez à Paul Ricœur et à son "identité narrative", qui reste soumise à un certain narrativisme. Il n'est en effet pas nécessaire de forcément passer par le récit pour se construire; d'autres genres peuvent être mobilisés (poésie, épistolaire, journal, essai). En outre, par la notion de style, vous montrez que le récit sur soi n'est jamais stabilisé, propre à produire une identité. Pensez-vous 
qu'il est nécessaire de travailler sur les différents discours avec les élèves, de les rendre "sensibles» à la puissance des possibles qui existent pour se comprendre? Peut-on alors se passer des règles des genres à un moment donné et à leur contexte historique?

MM: Je suis heureuse que ce point vous intéresse, car il m'a aussi beaucoup importé. Mon intérêt pour le style, la phrase, la syntaxe m’a en effet conduite à entrer en débat avec la pensée littéraire de Ricœur, qui trouvait dans la littérature un appui considérable pour comprendre la constitution des identités dans le temps. Ricœur a imposé une fois pour toutes l'évidence du caractère médiateur des formes: tout son travail sur l'interprétation a permis de montrer que nos expériences esthétiques sont faites pour aboutir dans notre monde, dans nos existences, parce qu'elles y jouent un rôle de modèles, de schèmes intermédiaires. Mais (et je dis tout cela beaucoup trop vite) Ricœur a aussi restreint cette fonction médiatrice à la seule forme du récit, en posant que l'identité du sujet dépend de sa capacité à raconter, et à se raconter, sa propre histoire. C’est une certaine idée du temps (un temps réparateur), une certaine morale, aussi, qui se donnent dans sa philosophie du sujet. Il m'a pourtant semblé qu'il fallait élargir cette considération des modes d'articulation entre les dispositifs esthétiques et les formes de la vie bien au-delà du cas du roman, et bien au-delà du modèle formel et moral de l'identité narrative. Je crois en effet qu'il existe une multitude de ressources, dans la littérature, pour ce que l'on pourrait appeler une herméneutique pratique.

C'est pourquoi je crois qu'il est important de travailler sur tous ces discours, et vous dites bien en quel sens: pour rendre les élèves sensibles aux possibles (à la multiplicité des possibilités de parole) qui existent pour... pour "se comprendre» certes, mais aussi, plus généralement, pour comprendre, pour dire quelque chose du monde, car la pratique du style n'est pas seulement une pratique de soi.

De ce point de vue, je ne crois pas qu'on doive abandonner l'idée de "genres», surtout si l'on accepte de ne pas en avoir une compréhension trop étroite, c'est-à-dire trop taxinomique. Bien plutôt, à nouveau, on peut tenter de rendre les élèves sensibles à "ce que peut un genre», à l'idée de vie qu'il favorise, à ce qu'une époque a pu y chercher. Pourquoi par exemple ne pas s'appuyer sur l'évolution des travaux narratologiques pour rendre les élèves sensibles à l'empire d'un certain genre de récits 
(le script) sur les imaginaires existentiels, commerciaux ou nationaux (ce qu'on appelle le storytelling) ? Ou leur parler de la difficulté de Sartre à écrire son autobiographie, lui qui ne voulait pas coïncider tout à fait avec un récit continu, avoir à s'y reconnaître?

Pour prendre un autre exemple: faire lire à de grands élèves «Le roman d'aventure» de Jacques Rivière, les aider à identifier le «nouveau plaisir " dont parle Rivière, lorsqu'il décrit ses contemporains comme des individus qui veulent être ceux "à qui quelque chose arrive», "sur qui un événement s'apprête à bondir ", les aider à comprendre ce que ce plaisir doit à Gide, et la façon dont il se retrouve dans les autres arts; les aider à distinguer cette façon de se tenir dans l'histoire d'autres rapports au temps, par exemple celui qu'engagent le roman proustien et les enjeux de la mémoire; les encourager à s'interroger sur ce que pouvait signifier cet espoir d'être quelqu'un à qui quelque chose arrive, qui espère une surprise et une déchirure... en 1913, alors que la catastrophe s'apprêtait en effet à venir, et que personne en réalité ne s'y préparait; bref, leur permettre d'acquérir cette sensibilité à l'histoire des formes et au sens que peuvent avoir des formes, voilà qui me semble constituer un enseignement intéressant.

RB: Je voudrais enfin vous interroger sur le statut de ces expériences esthétiques évoquées par vos "grands lecteurs", qui sont aussi de "grands écrivains». En me basant sur les travaux de Dominique Maingueneau et de Jérôme Meizoz, je me pose la question du statut de ces témoignages qui sont, d'une part, évidemment "scénographiés" (comme tout discours littéraire, ils font l'objet d'une mise en scène) et qui, d'autre part, concourent à la construction d'une "posture auctoriale», c'est-à-dire permettent à l'auteur de se positionner stratégiquement dans le champ littéraire. Cela pose non seulement la question de l'honnêteté de ces témoignages, mais aussi, et surtout, celle de leur statut par rapport aux expériences "ordinaires». En d'autres termes, ces témoignages qui évoquent des lectures "extraordinaires» nous apprennent-ils quelque chose au sujet de l'expérience commune que chacun d'entre nous peut faire de la littérature?

MM : Il y a beaucoup de choses dans votre question, qui est passionnante, et qui a des implications considérables pour les sujets qui m'intéressent; elle va peut-être me permettre de préciser ma position sur la valeur et 
le sens que l'on peut accorder aux formes de la pratique. Vous soulevez en fait deux choses: un doute à l'égard du caractère "représentatif", pour le dire vite, des textes que j'ai convoqués (textes d'écrivains, ou d'intellectuels, qui seraient pour cela très éloignés de l'expérience générale); et le sentiment que les écrits d'un «auteur" peuvent toujours faire l'objet d'une analyse en termes de "posture", de fabrique de soi. Vous présentez là deux raisons de se méfier de ces «terrains" que j’ai choisis pour mener mon analyse: d'une part parce qu'ils seraient inexemplaires (socialement inexemplaires) au regard de la vie ordinaire, d'autre part parce qu'ils seraient fabriqués, scénographiés, essentiellement stratégiques (socialement stratégiques, orientés vers le désir d'occupation d'une place).

Je suis sensible à la démarche sociologique qui sous-tend votre question, et je veux l'être, mais je me sens en désaccord au moins partiel avec ces deux directions: avec l'idée homogène qu'elles proposent des pratiques, et avec leur superposition.

J'estime beaucoup, et sans réserve, les travaux de Jérôme Meizoz, mais je crois qu'il ne faut pas les utiliser pour les faire répondre à une question qui n'est pas la leur; Jérôme Meizoz pose une question précise et circonscrite, celle de la fabrique d'un certain genre de singularité: la figure de l'écrivain sur la scène littéraire, et il y répond par la notion unifiante, et effectivement indépassable, de posture. Mais je ne crois pas qu'il fasse de cette fabrique d'une figure publique une allégorie du façonnement du sujet social dans sa globalité. En tout cas je combattrais vigoureusement cette idée si c'était le cas! En l'occurrence, la "posture», qui est effectivement l'essentiel lorsque l'on s'intéresse aux images et aux jugements échangés sur une scène de visibilité, ne m’apparait que comme un aspect, comme une valeur, comme une direction possible du sens que peuvent prendre l'ensemble des formes de la pratique sociale; elle s'insère dans une certaine région du social (la question de la scène justement, de l'espace de circulation des jugements, d'un certain rapport à la renommée ou à la célébrité, etc.). Mais tout ce qu'il entre de formes (de gestes, de rythmes, d'éthos) dans une vie, et même dans une vie d'écrivain, n'est pas épuisé par cette dynamique; un geste, par exemple, n'est pas toujours une image adressée en vue d'une reconnaissance, ou l'appropriation d'un code à l'intérieur d'un processus public de classement; il est aussi, par exemple, le site d'exercice d'une capacité (les textes de Mauss, de ce point de vue, sont loin de déboucher sur la définition de l'habitus 
que donnera Bourdieu); initialement, l'éthos ne désigne d'ailleurs pas une image, un mode de présentation de soi, mais, indissociablement, une manière d'être et un habitat; et cela en durcit un peu les enjeux que de les rapporter à l'idée d'un "positionnement stratégique", qui serait volontaire et maîtrisé (ce que Bourdieu lui-même n’a jamais formulé aussi unilatéralement).

En bref, la pensée de la posture s'intéresse dans l'écrivain à l'êtreécrivain, ou plutôt à la fabrique du paraître-comme-écrivain, paraître comme écrivain sur la scène littéraire (un paraître dont Jérôme Meizoz montre qu'il n'est pas un faire semblant, mais désigne le fait, considérable, d'avoir nécessairement à comparaître sur la scène du jugement littéraire, sur une scène où ce sont des évaluations qui s'échangent). Mais il ne me semble pas que tout ce qu'écrit un écrivain, a fortiori tout ce qu'il vit, mette en jeu en lui le fait d'être écrivain, et soit épuisé par un commentaire sur la façon dont il est écrivain, c'est-à-dire sur la manière dont il paraît comme écrivain sur cette scène du jugement littéraire. Ce n'est pas de ma part un désir de "protéger" la littérature des stratégies, c'est une conviction plus large sur les configurations individuelles et sociales: je ne crois pas que ce qu'il y a de formes (de gestes, d'images) dans la vie soit épuisé par cette dynamique de posture, de distinction, d'occupation d'une place dans la topographie sociale; je crois qu'il s'y engage aussi beaucoup d'autres valeurs, et d'autres liens; c'est d'ailleurs le sens de la stylistique ouverte de l'existence que je vise.

Je ne me suis donc pas intéressée chez Sartre, Bourdieu ou Michaux à la façon dont leur pratique des formes débouchait sur une disposition à écrire et sur une façon d'occuper, plus ou moins confortablement, plus ou moins originalement, la place de l'écrivain ou de l'intellectuel, et d'avoir une manière de l'occuper; il m'a semblé qu'ils avaient quelque chose de plus ample à dire sur la forme des pratiques (sur le rapport au monde sensible, aux rythmes, à autrui, sur l'effet d'une ouvre, etc.), et que je pouvais en cela créditer leurs textes d'une capacité descriptive. Je n'ai pas cherché à savoir si ces configurations descriptives, qui sont aussi des configurations existentielles, étaient authentiques ou artificielles, sincères ou stratégiques (cette question me semble piégée par ses termes mêmes, un peu comme l'hésitation entre authenticité et aliénation chez Rousseau). Je les ai en fait regardées comme des espaces de stylisation, des lieux où un sujet fait déjà quelque chose avec les formes ou avec 
les œuvres qui l'ont affecté, fait quelque chose qui revient à intervenir, même modestement, sur ses modes d'être.

Les textes que j'ai mobilisés ne sont d'ailleurs pas des textes sur la lecture, encore moins des discours tenus sur la littérature; ce sont, encore une fois, des configurations d'expérience, des configurations où l'expérience a pris forme, et où j'ai cru pouvoir identifier l'effet d'une lecture, la trace du passage de telle ou telle expérience esthétique dans une vie, dans cette vie-là, avec ses coordonnées et ses formes. Ce ne sont pas des textes de définition ou de déclaration de ce qu'il faudrait faire avec les œuvres, ce sont les manifestations d'une épreuve esthétique: l'épreuve traversée par ce sujet-là avec cette œuvre-là. En ce sens, ils me semblent avoir une vraie dimension d'exemplarité. Car si nous ne partageons pas avec les écrivains (sauf pour les artistes parmi nous) l'aventure de l'écriture, en revanche nous partageons avec eux la tâche de vivre, et c'est à cela que je me suis intéressée en eux: parce qu'ils disent quelque chose des enjeux du vivre, et parce qu'ils s'efforcent non seulement de le penser, mais aussi de le formuler, autrement dit parce qu'ils nous donnent des phrases pour re-phraser notre propre expérience. Ce serait dommage de se priver de cette immense réserve de pensées, au prétexte que les écrivains ne nous parleraient que de leur être-écrivain, ce que je ne crois pas du tout. Et ce serait plus étrange encore de se méfier de la littérature parce qu'elle risque des formes; c'est à vrai dire tout l'enjeu de mon travail: je crois que la littérature est «à transmettre» précisément parce qu'elle engage des formes, et en elles des pensées; des pensées précises, des pensées effectives, des pensées partageables.

C'est pour cela aussi que j'ai plus volontiers saisi mes individus là où ils se trouvaient en difficulté, mis au pied du mur, mal capables de rapatrier une expérience esthétique dans l'existence, comme je l'ai déjà rappelé. Je me suis par exemple penchée sur les différentes façons qu'avait eues Bourdieu de faire usage de la poésie, ou les différentes façons qu'avait eues Sartre de se conduire avec les œuvres d'art, ses manières de s'engager dans une expérience des romans ou des poèmes, de chercher à les établir dans le monde de l'action, mais aussi de ne pas y parvenir, et j'ai essayé de suivre en cela l'aventure complète d'une personnalité, en saisissant les effets de la lecture à l'échelle d'une vie tout entière. C'est l'occasion d'observer que la lecture est une pratique où un individu peut prendre en charge, de livre lu en livre lu, sa propre vie, les formes et les rythmes de sa vie. En l'occurrence, dans le cas de Sartre, les lectures 
successives sont l'occasion de se construire comme être dans le temps. Et toutes ne sont pas des appuis, loin s'en faut; certaines le mettent en grande difficulté; ce sont ces difficultés qui m’ont intéressée, et non pas ce que l'écriture de Sartre ou sa place dans la vie littéraire pourraient devoir à sa propre expérience des œuvres. Dans ce genre d'analyse, Sartre est sans doute une grande intelligence, mais pas, au sens où vous le dites, un «grand écrivain».

Pour autant, même si je ne m'accorde pas aux présupposés de votre question quant à la vision peut-être trop unifiante de tout ce qui s'échange sur la scène sociale, je ne veux pas esquiver ce qui probablement la motive, car je suis très sensible à la question de l'accès aux livres et de l'inégalité des trajectoires, qui vous occupe aussi. Mais justement, je ne crois pas qu'il faille se défendre de la force des textes littéraires comme on aurait à se défendre d'un dispositif coercitif; un texte n'est pas, sauf cas particulier, un dispositif d'assujettissement, je crois que c'est une puissance, une puissance appropriable, qui indique un rapport bien différent à la force. Il me semble que c'est la conscience de cette puissance qui peut faire l'objet de l'enseignement.

Marielle MACÉ

Centre de recherche sur les arts et le langage (CNRS-EHESS)

Raphaël BARONI

Université de Lausanne

Antonio Rodriguez

Université de Lausanne 